\title{
Propeller Selection by Means of Pareto Optimal Sets Applied to Flight Performance
}

\author{
Svatomir Slavik ${ }^{1}$, and Jan Klesa ${ }^{1, *}$ \\ ${ }^{1}$ Czech Technical University, Faculty of Mechanical Engineering, 16607 Praha 6, Czech Republic
}

\begin{abstract}
Selection process of the propeller for short take-off and landing (STOL) category aircraft is described. The aim is to achieve the highest possible performance with fixed propeller. The requirements are following: high maximal horizontal and cruise speeds, short take-off, high rate of climb and fixed propeller. These requirements are contradictory and so Pareto sets were used in order to find the optimal propeller. The method is applied for a family of geometrically similar propellers that are suitable for $73.5 \mathrm{~kW}(100 \mathrm{hp})$ piston engine designed for ultralight category aircraft with maximal take-off weight $472.5 \mathrm{~kg}$. The propellers have from two to eight blades, blade angle settings from $15^{\circ}$ to $40^{\circ}$ and diameter from $1.1 \mathrm{~m}$ to $2.65 \mathrm{~m}$. Pareto frontier is designed for each couple of parameters and the optimal propeller is selected according to these results. For comparison, the optimal propeller selection from the propeller family by means of a standard single-optimal process based on the speed power coefficient $c_{s}$ is also used. Using of Pareto sets leads to considerable performance increase for the set of contradictory requirements. So, high performance power system with low price (fixed pitch propeller) for STOL category aircraft can be designed. The described method can be used for the propeller optimization in similar cases.
\end{abstract}

\section{Introduction}

An optimal propeller for a designed aeroplane with a specified engine represents in the general case its optimal design that means assessment of such propeller blade geometry and number of blades that ensure fulfilment of required flight performance of the aeroplane according to a design aeroplane strategy. Such access to the optimal power unit belongs to propeller producers.

Aeroplane designers and producers, designing a propulsion, often proceed from a selection of a suitable propeller from a family of geometry similar propellers with different number of blades, pitch blade angles and diameters that are fit for the power engine of the aeroplane. The optimal design of a power unit so switches to an optimal selection task. The classic optimal selection of the optimal propellers uses is based on the speed power coefficient. This procedure pick out such propeller geometry from the propellers family that insures the maximum propeller efficiency for chosen engine working point (power and speed) at a design flight speed. This selection is only one point optimal procedure

* Corresponding author: jan.klesa@,fs.cvut.cz 
regardless of different flight performance. The selection is bound to flight performance only by the designed flight speed and engine mode. The full-value optimal selection is based on optimization criteria from. Minima and maxima of flight performance are taken as the optimization criteria.

One of the possible approaches to the optimizing selection represents the multiobjective optimization method by means of Pareto optimal sets [1, 2]. Flight performance corresponds to objective functions with a propeller as a variable. Determination of optimal fronts in Pareto sets, in this case optimal fronts of elected pairs of objective functions (optimal fronts of elected performance flight), particularised the optimal selection of propellers. All elements of the optimal Pareto fronts are optimal solution because it cannot be determined from the principle of contradictory requirements which propeller is better. The selection of one optimal propeller depends on preference (weights) of the individual flight performance extremes or other (non-performance) economic and operating criteria as price, availability, reliability and others.

\section{Methods}

\subsection{Pareto sets of flight performance for optimization selection of propellers}

In the optimization selection of propellers from the family of propeller, the discrete points represent individual propellers. If the optimization criteria require extremes of flight performance, then the propellers are defined by their geometry for determination of propeller aerodynamic characteristics. The propeller geometry comprises both continuously geometric parameters (mainly distribution of the chord length, twist and thickness of the used airfoils along the blade) and discrete parameters - the propeller diameter, number of blades.

The propeller aerodynamic characteristics represent the dependence of the thrust and power coefficients on the advance ratio $\left(c_{T}(\lambda), c_{N}(\lambda)\right)$ that together with an engine power curve enables to set the available isolated thrust curve of the power unit. The thrust curve for the adequate power engine regime of the flight performance together with the respective aerodynamic characteristics of the aeroplane and physical constants of the flight environment present input parameters for the determination of the performance objective function. The interaction of propeller and aeroplane takes into account the effective propeller thrust and the drag curve of the aeroplane is so taken without a propeller stream influence on the drag.

Pareto sets are composed from final number of points (equal to the number of propellers in the family of propeller) of appropriate pairs of the contradiction performance objective functions: [maximum horizontal flight speed - take-off distance], [maximum horizontal flight speed - maximum rate of climb], [take-off distance - maximum rate of climb]. The pair of [maximum horizontal flight speed (continuous engine regime) - maximum horizontal flight speed (cruise regime)] is also included.

The optimization selection of the propeller by means of Pareto optimal sets consists of the following sequential actions:

1. Assessment of free flight aerodynamic characteristics of the aeroplane (lift curve $c_{L}(\alpha)$, lift-drag polar $c_{L}\left(c_{D}\right)$ without the propeller influence on the aeroplane drag in the relevant flight configuration of the particular flight performance [3-6].

2. Determination of propeller aerodynamic characteristics - thrust and power coefficients $c_{T}(\lambda), c_{N}(\lambda)$. 
3. Calculation of the isolated thrust curve $T_{i s}(V)$ corresponding to the particular flight performance with the competent engine regime, $T_{i s}(V)$ correction to the true $T(V)$ and effective thrust $T_{e f}(V)$.

4.Calculation of all flight performances with the free aeroplane aerodynamic characteristics corrected for the respective ground effect of each flight performance.

5. Set up of the contradiction pairs of the flight performance and creation of Pareto set graphs.

6.Evaluation of optimal Pareto fronts on Pareto sets graphs as the optimization selection.

\subsection{Aerodynamic characteristics of the model aeroplane}

A model study of a small two-seat sport aeroplane with a requirement for a short takeoff and landing (STOL) was chosen. The aeroplane is designed as the high-wing arrangement with $13 \mathrm{~m}^{2}$ trapezoidal wing of the aspect ratio equals to 7.2. The wing is equipped with a combined high-lift device on the leading and trailing edge - a slotted leading edge (slat) and Fowler flap. The aeroplane is drawn up with the standard side-byside seat arrangement and a fixed taildragger landing gear with fairing.

Lift and drag aerodynamic characteristics were determined according to the methodology [3] and [4] for the following flight lift device configuration:

1. Take-off lift configuration - slat + Fowler flap $15^{\circ}$.

2. Cruise configuration - retracted lift devices.

The primary characteristics are determined without the propeller influence on the aerodynamic drag of the aeroplane and without the ground effect. The characteristics correspond to moment-balanced states, in the case of landing to the front centre of gravity. The primary lift and drag characteristics of take-off and landing configurations are corrected for the ground effect depending on the actual height above the ground. An additional aeroplane aerodynamic drag due to the propeller stream is included in the true propeller thrust.

\subsection{Powerplant}

The ROTAX 912 Aircraft Engine has been selected. The engine, primary designed for this category of aeroplanes, has take-off power of $73.5 \mathrm{~kW}$ and is equipped with a propeller speed reduction unit with gear ratio $\mathrm{i}=2.43$. The take-off regime corresponds to a maximum power at maximum permissible speed for a short-term use - maximum 5 minutes, the continuous regime is $90 \%$ of the take-off power at reduced engine speed without any time limit and the cruise regime (economic engine operation) means $75 \%$ of the continuous regime.

\subsection{Propellers}

\subsubsection{Propeller Family}

The propeller family is created as geometrical similar propellers of a three-blade propeller that is fit for $73.5 \mathrm{~kW}(100 \mathrm{hp})$ power engine. The three-blade propeller was extend to other numbers of blades: two-blade, four-blade, five-blade, six-blade and eightblade propeller with eleven pitch blade angles from $15^{\circ}$ to $40^{\circ}$ at $75 \%$ of radial distance. Range of propeller diameter of the total propeller family was from $1.1 \mathrm{~m}$ to $2.65 \mathrm{~m}$ but each number of blades for a given pitch blade angle is only a part of this range. Extremely 
overloaded propeller that reaches the permissible engine rotational speed practically at the beginning of the take-off limits the smallest diameter. The maximum diameter represents very lightly loaded propeller and is demarcated by unacceptably extend the take-off and simultaneously decreases rate of climb.

\subsubsection{Aerodynamic characteristics of propellers}

Aerodynamic characteristics of propellers needed to determine thrust curves are dependencies of the thrust and power coefficients on the advance ratio, i.e. $c_{T}(\lambda)$ and $c_{N}(\lambda)$. The vortex blade theory of an isolated propeller is used. Free helix vortex surfaces with a constant pitch leaving the boundary vortex of each blade generate a field of the induced velocities that adjust the magnitude and direction of free flow along the propeller blade. The aerodynamic forces acting along the blade so can be considered as two-dimensional airfoil characteristics with the incoming free flow corrected to the induced angle of attack. The calculations of the propeller aerodynamic characteristics were performed using the numerical model $[7,8]$. Input geometric data involve, except diameter D and number of blades $\mathrm{z}$, also the distribution of the chord length, twist and thickness of the used airfoils along the blade. The calculation was done for every blade pitch setting and number of blades.

\subsection{Thrust curves of the power unit}

The thrust curve of the power unit has the meaning of the available thrust in dependence on flight speed. First, the thrust curves of the isolated propellers were calculated and then these isolated thrusts were corrected for influence of the installation. The corrected thrust represents the true thrust. Because the additional drag of the aeroplane (both friction and pressure part) due to the increased speed of the propeller stream is thus dependent on the propeller thrust, it is preferable for the calculations of the flight performance to introduce so-called effective thrust. The effective thrust is the true thrust reduced by the additional propeller drag. The drag curve of the aeroplane without the additional propeller drag thus remains the same for all alternatives of the propeller propulsion units. The friction component depending on the thrust and wetted area influenced by the propeller flow was evaluated according to literature [6].

\subsection{Flight performance for Pareto sets}

Pareto sets require the pairing of such flight performances, which are contradictory. The respective pairs of flight performance required to achieve their extreme values (minimum, maximum) act on each other so that increasing one flight performance towards the extreme decreases the second flight performance from its desired extreme.

In general, it can be expected that the propellers with good take-off performance are not good for reaching maximum flight speeds and vice versa. Therefore, for pairs of the flight performance for Pareto set, the following are used:

1. Maximum horizontal flight speed (continuous engine regime) - Take-off distance (take-off engine regime)

2. Maximum horizontal flight speed (cruise engine regime) - Take-off distance (take-off engine regime)

3. Maximum horizontal flight speed (continuous engine regime) - Maximum rate of climb (take-off engine regime)

4. Maximum horizontal flight speed (cruise engine regime) - Maximum rate of climb (take-off engine regime) 
5. Take-off distance (take-off engine regime) - Maximum rate of climb (take-off engine regime).

6. Maximum horizontal flight speed (continuous engine regime) - maximum horizontal flight speed (cruise regime)

\section{Results}

Pareto sets of six-selected flight performance pairs discussed in the section 2.7 are plotted in Fig. 3 to 8. For clarity, only limited areas near Pareto optimal fronts from the all propeller family combinations 3 are depicted. The broken line connects points form Pareto optimal fronts. Selection by means of speed power coefficient $c_{s}$ is used for comparison (see cs opt points in Figs. 1 to 6).

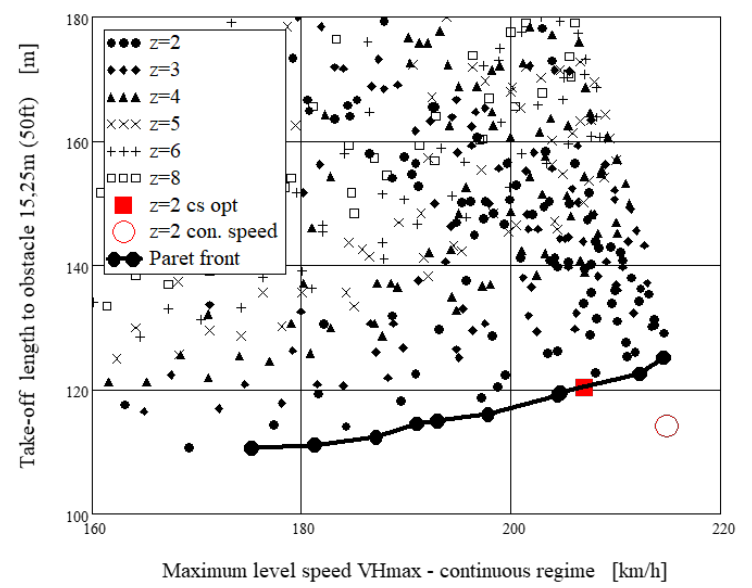

Fig. 1. Pareto frontier for maximum horizontal speed (continuous engine regime) and take-off distance.

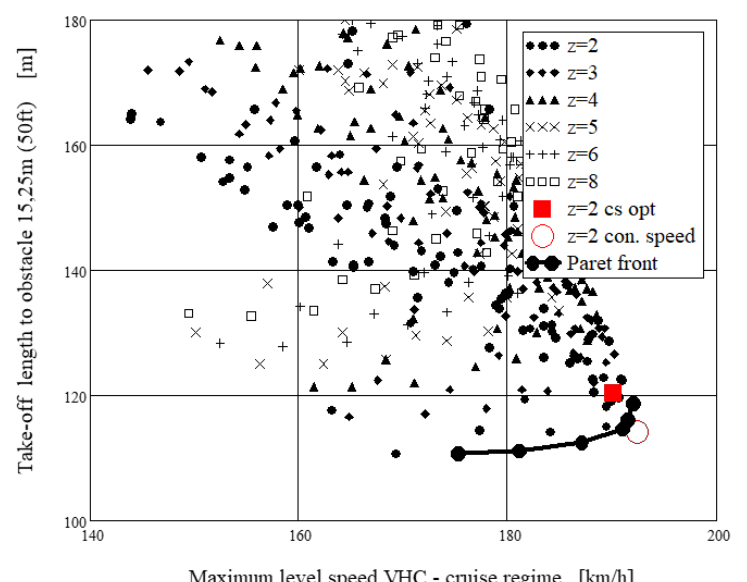

Fig. 2. Pareto frontier for maximum horizontal flight speed (cruise engine regime) - takeoff distance. 


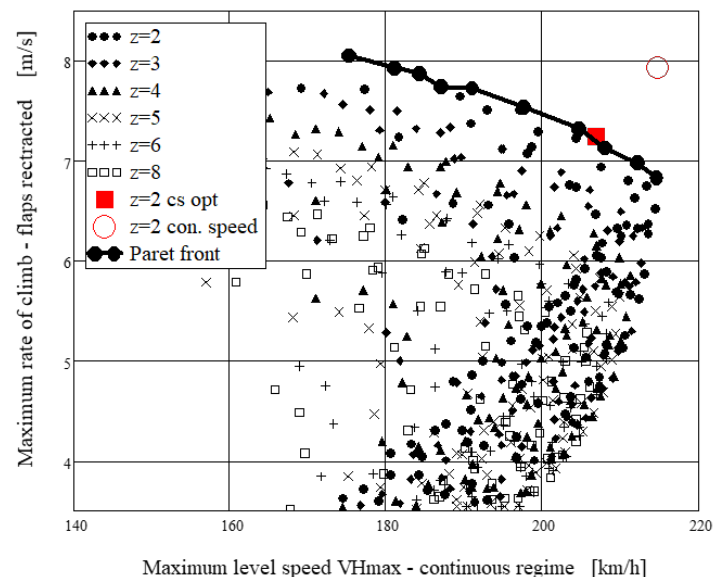

Fig. 3. Pareto frontier for maximum horizontal flight speed (continuous engine regime) and maximum rate of climb (take-off engine regime).

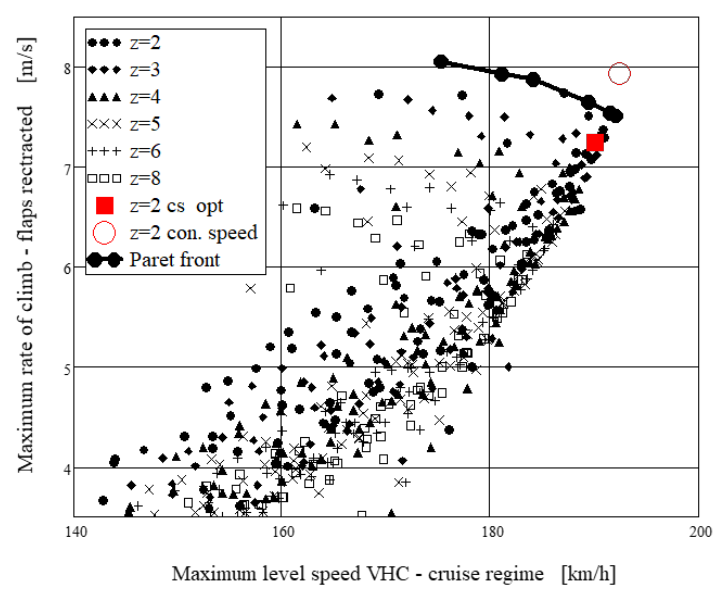

Fig. 4. Pareto front for maximum horizontal flight speed, (cruise engine regime) and maximum rate of climb (take-off engine regime). 


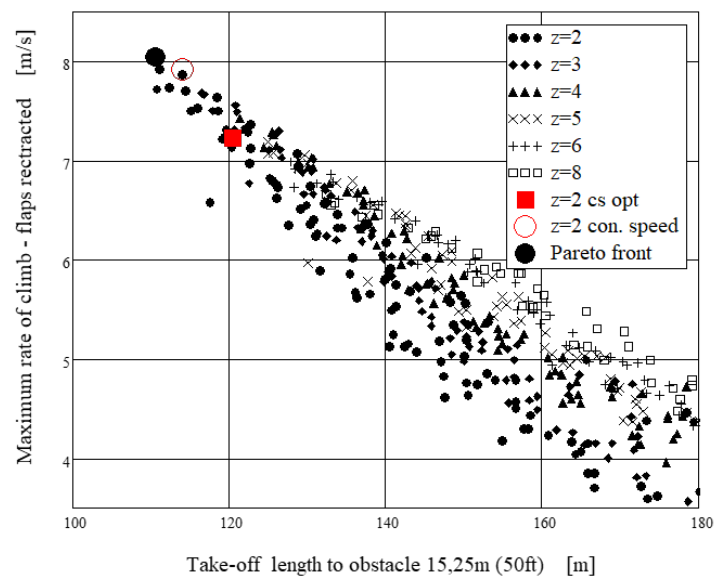

Fig. 5. Pareto frontier for take-off distance (take-off engine regime) and maximum rate of climb (take-off engine regime).

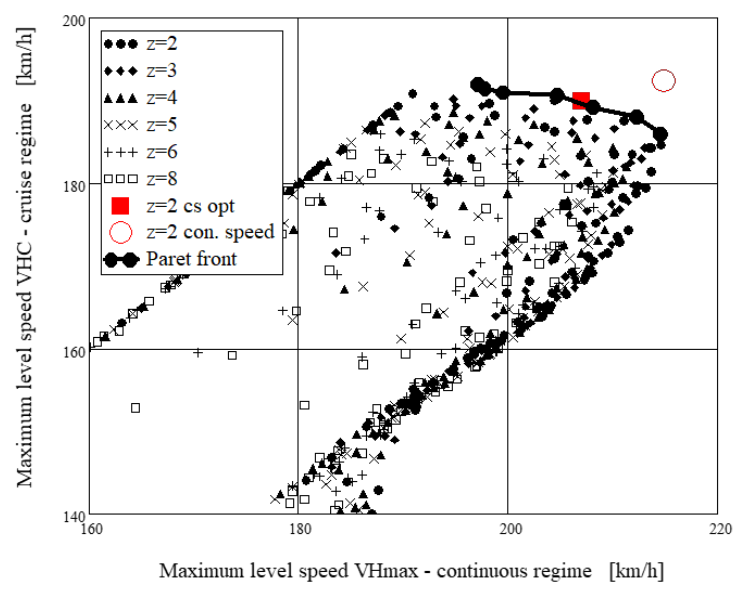

Fig. 6. Pareto frontier for maximum horizontal flight speed (continuous engine regime) and maximum horizontal flight speed (cruise regime).

\section{Conclusion}

Multi-objective optimization using Pareto sets of flight performance of $473 \mathrm{~kg}$ small STOL sport aeroplane powered by $73.5 \mathrm{kw}(100 \mathrm{hp})$ engine to select a fixed propeller from a family of geometrical similar propellers was performed. The propeller family included propellers from two-blade to six-blade and eight-blade each propeller for 11 pitch blade angles from $15^{\circ}$ to $40^{\circ}$ and diameters range from $1.1 \mathrm{~m}$ to $2.65 \mathrm{~m}$.

The optimization criteria required maximum level speed, maximum rate of climb and minimal take-off. The optimal Pareto fronts were investigated for four pairs of opposing flight performance:

1. Maximum horizontal flight speed - Take-off distance

2. Maximum horizontal flight speed, - Maximum rate of climb

3. Take-off distance - Maximum rate of climb.

4. Maximum horizontal flight speed - maximum horizontal flight speed (cruise regime). 
Length of take-off path and maximum rate of climb relate to the take-off engine power regime, maximum horizontal flight speed was considered for both continuous and cruise power regime. Pareto optimal study leads to the following conclusions:

1. Only two-blade propellers belong to all Pareto optimal fronts.

2. One cut-off point of Pareto optimal fronts for maximum horizontal speed corresponds to $\mathrm{D}=1.95 \mathrm{~m}$, pitch blade angle $\phi_{0.75}=22.5^{\circ}$ for the continuous engine regime and to $\mathrm{D}=2 \mathrm{~m}$, $\phi_{0.75}=20^{\circ}$ for cruise regime.

3. The opposite cut-off point of Pareto optimal front for both maximum rate of climb and minimal take-off distance corresponds one propeller: $\mathrm{D}=2.1 \mathrm{~m}$, pitch blade angle $\phi_{0.75}=15^{\circ}$.

4. The constant speed propeller achieves better performance than the best performance of the fixed pitch and ground adjustable propellers for all four optimization criteria.

Pareto Multi-objective optimization meet fixed pitch two blade propellers with diameter range increasing from $\mathrm{D}=1.95$ to $2.2 \mathrm{~m}$ with a corresponding decreasing of pitch blade angle range from $\phi_{0.75}=22.5^{\circ}$ to $12.5^{\circ}$.

To compare Pareto multi-objective optimization with standard selection propeller from a family of geometrical similar propellers by means the speed power coefficient the design was performed for design speed from middle of Pareto optimal front of maximal horizontal speed for continuous engine regime. The single-mode optimization selection for two-blade propeller confirms Pareto optimization selection: diameter $\mathrm{D}=2.015 \mathrm{~m}$ and pitch blade angle $\phi_{0.75}=20^{\circ}$. The speed power coefficient methods is limited by single-regime design given by flight speed and engine regime and the design clearly not tied to flight performance

Two blades propeller of the family propeller considered as a constant speed propeller with diameter obtained by the speed power coefficient presents an increase in flight performance limits of the fixed pitch propeller.

This work was supported by the EU Operational Programme Research, Development and Education, under the Centre of Advanced Aerospace Technologies, project No. CZ.02.1.01/0.0/0.0/16_019/0000826, Faculty of Mechanical Engineering, Czech Technical University in Prague.

\section{References}

1. K. Deb, Multi-Objective Optimization Using Evolutionary Algorithms (Wiley, 2001)

2. K.C. Tan, E.F. Khor, T.H. Lee, Multiobjective Evolutionary Algorithms and Applications (Springer, 2005)

3. E. Torenbeek, Synthesis of Subsonic Airplane Design (Delf University Press, Delft, 1982)

4. D.P. Raymer, Aircraft Design: A Conceptual Approach (AIAA, 1989)

5. J.T. Lowry, Performance of Light Aircraft (AIAA, 1999)

6. J.J. Ruijgrok, Elements of Airplane Performance (Delft University Press, Delft, 1990)

7. V.L. Alexandrov, Vozdushnye vinty (Airscrews) (Oborongiz, Moscow, 1951)

8. J. Klesa, AIAA 2014-3132 (2012)

9. V. Daněk, Mechanika letu I - letové výkony (CERM, Brno, 2009)

10. J. Salga, J. Švéda, Mechanics of Flight I (Perfomance) (Military Academy, Brno, 1990)

11. B.W. McCormick, Aerodynamics, Aeronautics, and Flight Mechanics (Wiley, 1979) 